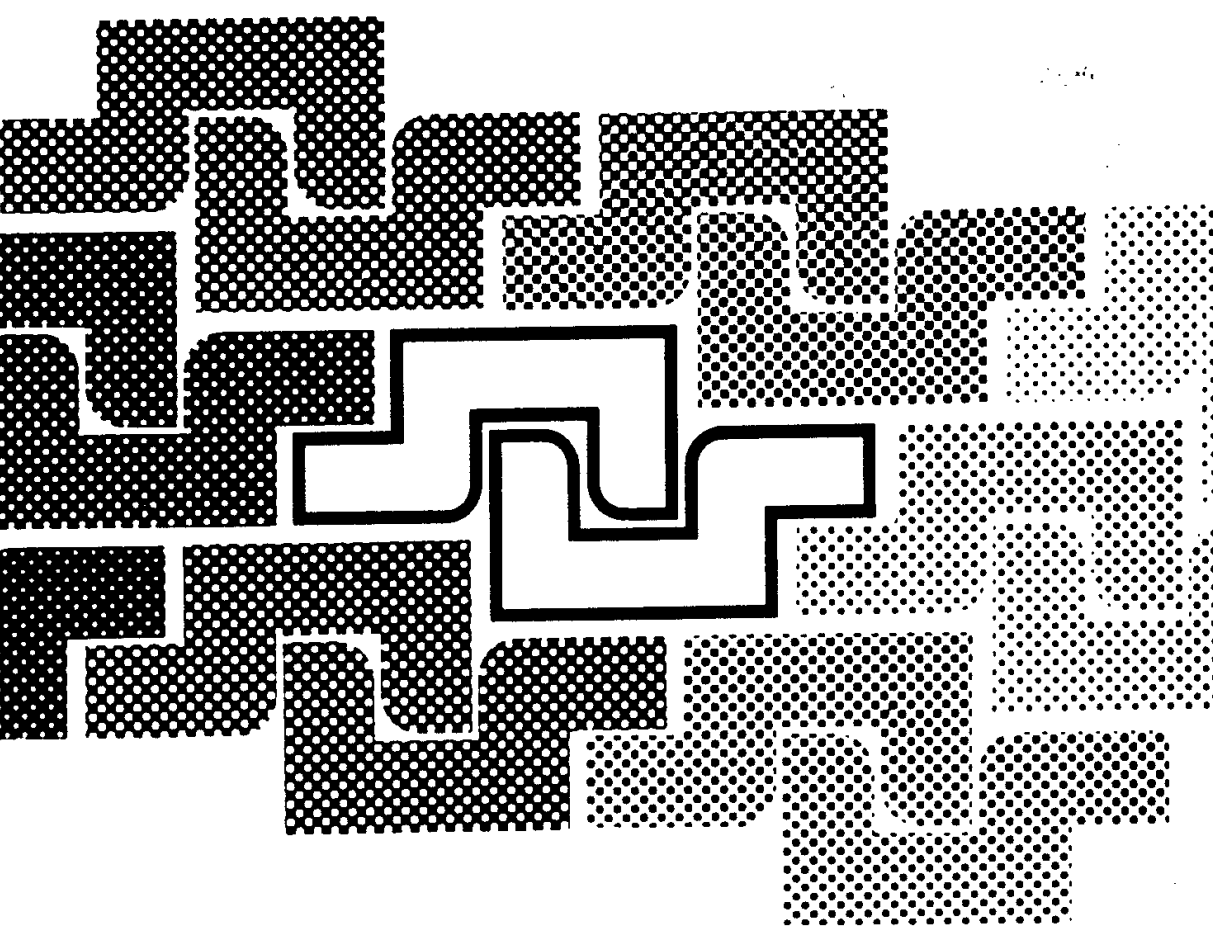




\section{6 \\ NATO ASI Series}

\section{Advanced Science Institutes Series}

A Series presenting the results of activities sponsored by the NATO Science Committee, which aims at the dissemination of advanced scientific and technological knowledge, with a view to strengthening links between scientific communities.

The Series is published by an international board of publishers in conjunction with the NATO Scientific Aflairs Division
A Life Sciences
B Physics

C Mathematical and Physical Sciences

D Behavioural and Social Sciences

E Applied Sciences

F Computer and Systems Sciences

G Ecological Sciences

H Cell Biology

I Global Environmental Change

\section{PARTNERSHIP SUB-SERIES}

1. Disarmament Technologies

2. Environment

3. High Technology

4. Science and Technology Policy

5. Computer Networking
Plenum Publishing Corporation

London and New York

Kluwer Academic Publishers

Dordrecht, Boston and London

\section{Springer-Verlag}

Berlin, Heidelberg, New York, London,

Paris and Tokyo

The Partnership Sub-Series incorporates activities undertaken in collaboration with NATO's Cooperation Partners, the countries of the CIS and Central and Eastern Europe, in Priority Areas of concern to those countries.

\section{NATO-PCO-DATA BASE}

The electronic index to the NATO ASI Series provides full bibliographical references (with keywords and/or abstracts) to more than 50000 contributions from international scientists published in all sections of the NATO ASI Series.

Access to the NATO-PCO-DATA BASE is possible in two ways:

- via online FILE 128 (NATO-PCO-DATA BASE) hosted by ESRIN,

Via Galileo Galilei, I-00044 Frascati, Italy.

- via CD-ROM "NATO-PCO-DATA BASE" with user-friendly retrieval software in English, French and German (@ WTV GmbH and DATAWARE Technologies Inc. 1989).

The CD-ROM can be ordered through any member of the Board of Publishers or through NATOPCO, Overijse, Belgium.

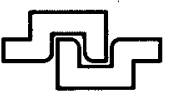

3. High Technology - Vol. 45 


\section{Optical Resonators - \\ Science and Engineering}

edited by

Ram Kossowsky

Emerging Technologies, Inc.,

Pittsburgh, Pennsylvania, U.S.A.

Miroslav Jelinek

Institute of Physics,

Czech Academy of Science,

Prague, Czech Republic

and

Josef Novak

Institute of Electrical Engineering,

Slovak Academy of Science,

Bratislava, Slovak Republic
SPC $97-1056$

F6170897-W 


\title{
BEAM DIVERGENCY STUDIES ON A LONG PULSE XECL EXCIMER LASER
}

\author{
R.M. HOFSTRA, F.A. VAN GOOR AND W.J. WITTEMAN \\ University of Twente \\ P.O. Box 217, 7500 AE Enschede, The Netherlands
}

Abstract.

A nearly diffraction limited output beam was obtained from a hard edge confocal positive branch unstable resonator. Improvement of the far field energy profile can be obtained by using partial reflecting hard edge outcoupling mirrors. Further improvement can be obtained by using gaussian mirrors.

\section{Introduction}

For the application field of our $1 \mathrm{kHz}, 1 \mathrm{~kW}$ long pulse $\mathrm{XeCl}$ excimer laser developed by the Nederlands Centrum voor Laser Research (NCLR) [1], the divergence of the optical beam is of utmost importance. The active medium of this laser has large discharge dimensions relative to the size of the fundamental gaussian mode of a stable plano-concave resonator. The beam from such a resonator is therefore multimode with a relatively poor divergence. With our system a $1.5 \mathrm{~m}$ long stable resonator consisting of a concave back mirror with a radius of curvature of $10 \mathrm{~m}$ and a flat partial reflector (50\% reflectivity) as outcoupling mirror resulted in a homogeneous, nearly square beam of $23 \mathrm{~mm} \times 22 \mathrm{~mm}$ having a pulse energy of approximately $500 \mathrm{~mJ}$ and a divergence of $7 \mathrm{mrad}$ (full angle). However a diffraction limited beam of this size would have a divergence of about $30 \mu \mathrm{rad}$. To decrease the divergence the number of oscillating modes should be decreased to, preferably, one fundamental mode with dimensions that fill the available gain volume. This can be done with an unstable resonator. For our system, having a low gain $\left(2-6 \% \mathrm{~cm}^{-1}\right)$ compared to the commercially available short pulse $\mathrm{XeCl}$ lasers and a long gain duration (approximately $250 \mathrm{~ns}$ ), a positive branch confocal unstable resonator is found to be the best choice (compare 


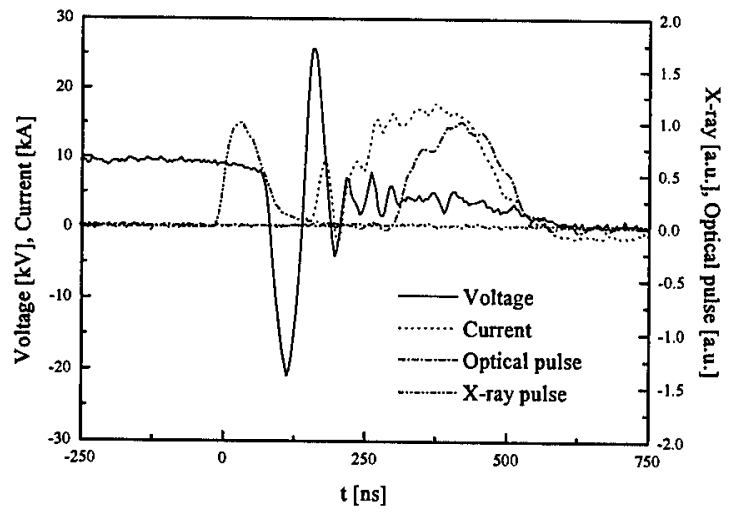

Figure 1. Typical waveforms of the discharge voltage, discharge current, x-ray preionisation pulse and optical output pulse

refs. $[2,3,4])$, with outcoupling through the convex mirror to get a collimated beam. In this paper we present a detailed experimental study of unstable resonators for a low gain, long pulse $\mathrm{XeCl}$ excimer laser.

\section{Experimental configuration}

The experiments have been performed with a XeCl laser system having a $2.5 \mathrm{~cm} \mathrm{x} 2 \mathrm{~cm} \times 60 \mathrm{~cm}$ (electrode distance $\mathrm{x}$ width $\mathrm{x}$ length) $\mathrm{x}$-ray preionised high pressure discharge[5]. The gas mixture during the experiments consisted of approximately $1 \mathrm{mbar} \mathrm{HCl}, 10 \mathrm{mbar} \mathrm{Xe}$ and $\mathrm{Ne}$ as a buffer gas up to a pressure of 5 bar. The discharge was excited by a spiker-sustainer circuit. The laser operates in resonant overshoot mode[6]. Figure 1 shows typical waveforms for the $\mathrm{x}$-ray preionisation pulse, discharge voltage and discharge current. The efficiency is maximal if the system operates under matched discharge conditions, i.e. if the voltage on the pulse forming network (PFN) equals twice the steady state voltage of the discharge. The output can be increased by increasing the PFN voltage but then the efficiency drops. If the PFN voltage is set too high the discharge becomes unstable and ends prematurely in streamers and arcs. To prevent electrode wear the system is operated under almost matched discharge conditions. The results presented in this paper are obtained under these conditions, unless stated otherwise.

The near and far field profiles are measured using a gated image intensified CCD camera. For the near field measurement the beam is transported 


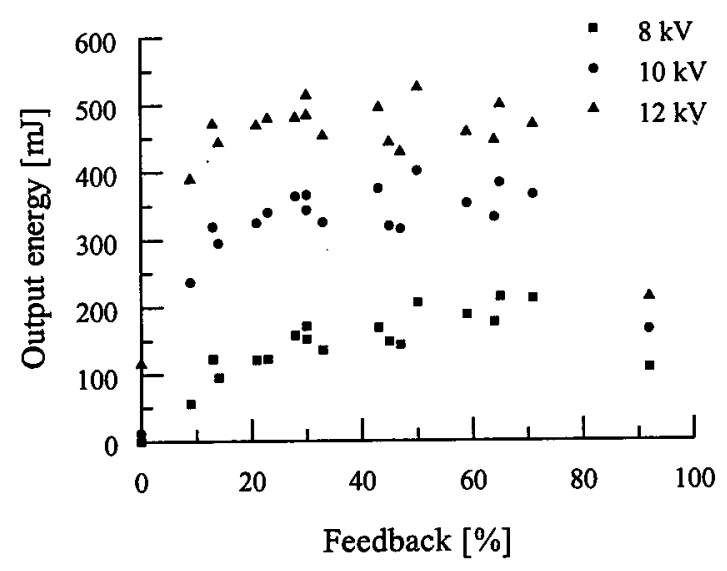

Figure 2. Output energy as a function of the geometrical feedback for different charging voltages of the sustainer

away from the laser with a relay imaging system consisting of two $50 \mathrm{~cm}$ focal length lenses placed $1 \mathrm{~m}$ apart. The focal plane of the first lens is just behind the outcoupling mirror to ensure that we have an exact image of the outcoupled field at the scintillator which is placed in de focal plane of the second lens. As a scintillator we use a thin film of a solution of sodium salicylate in water between two suprasil windows. To ensure that the response of the scintillator remains linear the beam is attenuated to about $1 \%$ of its energy. The CCD camera we used showed some nonlinear response which was found to be related to the CCD and not to the image intensifier. Hence a correction can be made for this nonlinear respons.

The focus field pattern is measured by focussing the attenuated beam (attenuation to about $0.05 \%$ ) on the scintillator using a concave mirror with a radius of curvature of $10 \mathrm{~m}$. To obtain the image the CCD camera has been equipped with a microscope objective.

\section{Stable resonators}

The simplest type of resonator is the stable resonator. Good energy extraction from the gain medium is can easily be obtained if proper feedback is used. Figure 2 shows the output from a plane-plane resonator as a function of the feedback of the resonator. For a stable resonator this feedback is solely determined by the product of the reflectances of the mirrors

$$
\gamma=R_{1} R_{2}
$$




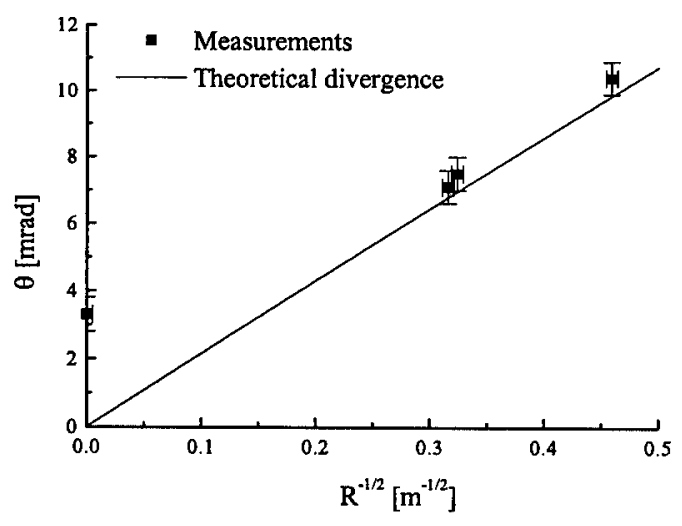

Figure 3. Divergence of the output beam from a stable resonator as a function of the radius of curvature of the rear mirror

Figure 2 shows that for a sustainer voltage higher than $10 \mathrm{kV}$ the output is more or less constant for a feedback between 20 and $70 \%$. If the feedback is far below $20 \%$ the outcoupling losses are larger than the gain, hence no output beam can be formed.

In our system where the discharge dimensions are much larger than the fundamental gaussian mode the output beam is multimode. This results for stable resonators, especially for stable plano-concave resonators in an output beam that is very homogeneous in the near field. From our $2.5 \mathrm{~cm} \times 2.0 \mathrm{~cm}$ discharge (height $\mathrm{x}$ width) we obtained homogeneous nearly square beams with a size of about $23 \mathrm{~mm} \times 22 \mathrm{~mm}$.

The problem with these stable resonators is their divergence. As the output beam is multimode the divergence is rather high in comparison with the diffraction limit. Figure 3 shows the divergence of different stable plano-concave resonators as a function of the radius of curvature of the rear mirror. In approximation the divergence is given by [7]

$$
\theta \approx \frac{D}{\sqrt{L R}}
$$

where $D$ is the dimension of one side of the beam aperture, $L$ is the length of the cavity and $R$ the radius of curvature of the concave rear mirror. It is seen in figure 3 that the experiments are in good agreement with the theory except for the plane-plane resonator but for this resonator the approximation is not longer valid. 


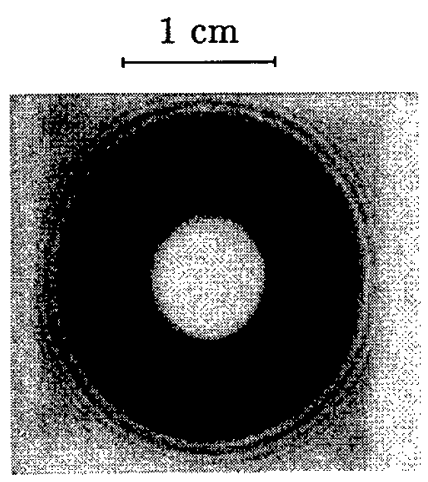

Near field

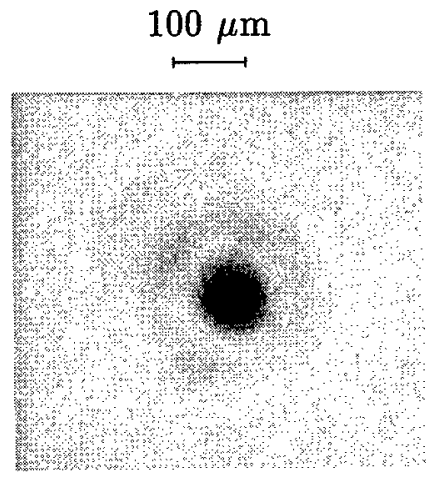

Far field

Figure 4. Near field profile (left) and focus profile (right) of the beam from a hard edge unstable resonator $(M=2.4)$ with a central HR spot of $8 \mathrm{~mm}$ diameter focussed by a $10 \mathrm{~m}$ radius concave mirror

\section{Hard edge unstable resonators}

To increase the size of the fundamental mode of the resonator one has to use an unstable resonator. An unstable resonator reaches this fundamental mode earlier if the magnification of the resonator is larger [8]. So the magnification ought to be chosen as large as possible within the constraints of the gain medium. In our system we found with the stable resonators a minimum feedback of $20 \%$. This minimum value of about $20 \%$ for the feedback determines the choice of the magnification of the unstable resonator which is given by

$$
M=-\frac{R_{2}}{R_{1}}
$$

where $R_{1}$ is the radius of curvature of the outcoupling convex mirror and $R_{2}$ is the radius of curvature of the concave rear mirror. The feedback of an unstable resonator is given by

$$
\gamma=\frac{1}{M^{2}}
$$

Thus we used an unstable resonator with a magnification of 2.4 to have enough feedback (18\%).

Hard edge unstable resonators are unstable resonators where the outcoupling mirror shows a step in the reflectance profile. This results in a step in the near field intensity distribution of the outcoupled beam. For symmetry reasons we used a centered reflecting spot on the outcoupling mirror, so that the outcoupled beam shows a cylindrical symmetry as shown in figure 4. Figure 4 shows also a typical hard edge unstable resonator focus profile: not only a central peak but also a ring structure. 


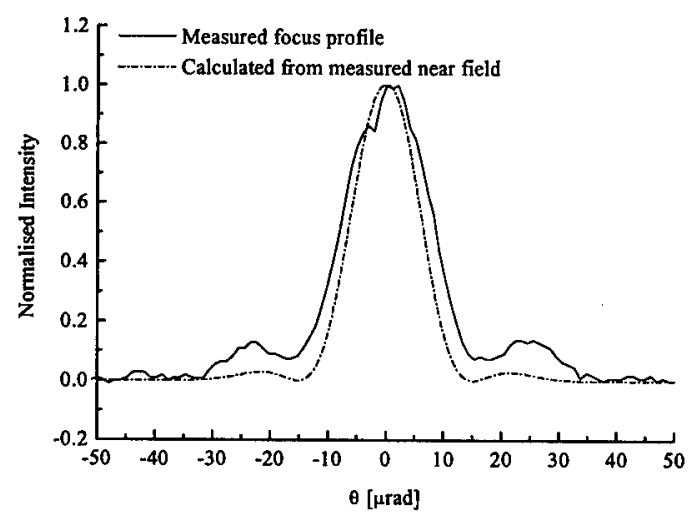

Figure 5. Comparison between far field energy distributions from theory and experiment

\subsection{HIGH REFLECTIVITY HARD EDGE UNSTABLE RESONATORS}

The beam profiles in figure 4 are obtained with a hard edge unstable resonator with a magnification $M$ of 2.4 and a central high reflectivity (HR) spot of $8 \mathrm{~mm}$ diameter. To determine the beam quality of this beam we calculated the Times Diffraction Limited (TDL) parameter [9]. This TDL parameter is based on the comparison of the measured focus energy distribution and a calculated focus energy distribution. This calculated focus energy distribution is derived from the measured near field energy distribution using a uniform phase distribution. Figure 5 shows these two far field energy distributions for the above mentioned resonator.

The TDL-parameter can now be determined using

$$
\mathrm{TDL}=\frac{\theta_{\text {meas }}}{\theta_{\text {calc }}}\left(\frac{k_{\text {calc }}}{k_{\text {meas }}}\right)^{\frac{1}{2}}
$$

where $\theta_{\text {meas }}$ and $\theta_{\text {calc }}$ are the measured and calculated beam divergence respectively, and $k_{\text {meas }}$ and $k_{\text {calc }}$ the respective energy content within that divergence angle. So the TDL parameter does not only compare focus sizes, but also contains the energy content. A beam with a value 1 for the TDL parameter is a beam that is diffraction limited, not only with respect to size but also with respect to energy.

The full divergence angle based on the first minimum is $32 \mu \mathrm{rad}$ for the measured beam and $30 \mu \mathrm{rad}$ for the calculated beam. The measured beam has approximately $60 \%$ of the energy within the central peak and the calculate beam $82 \%$ of the energy. This results in a TDL-parameter 
TABLE 1. Characteristics of and experimental results obtained with the laser equipped with different hard edge unstable resonator configurations and operated under matched discharge conditions

\begin{tabular}{cccccccc}
$M$ & $R[\%]$ & $D_{r}[\mathrm{~mm}]$ & $\gamma[\%]$ & $\theta_{d}[\mu \mathrm{rad}]$ & $E[\mathrm{~mJ}]$ & $\tau[\mathrm{ns}]$ & $k[\%]$ \\
\hline 2.4 & 100 & 8 & 17.7 & 32 & 339 & 160 & 60 \\
2.4 & 72 & 8 & 12.5 & 37 & 334 & 146 & 70 \\
2.4 & 45 & 8 & 7.8 & 31 & 305 & 146 & 50 \\
2.0 & 72 & 10 & 17.5 & 36 & 362 & 149 & 70 \\
1.6 & 45 & 12 & 17.5 & 34 & 359 & 160 & 30
\end{tabular}

of 1.3 for our beam. Thus we reached almost the optimal result that can be obtained with this resonator. However, about $40 \%$ of the energy is lost in side lobes. This loss of energy in side lobe(s) can be decreased by using partially reflecting hard edge outcoupling mirrors.

\subsection{PARTIAL REFLECTIVITY HARD EDGE UNSTABLE RESONATORS}

Table 1 shows the characteristics of different hard edge unstable resonators used in the experiments. $R$ in table 1 is the reflectivity of the reflecting area and $D_{r}$ its diameter. The geometrical feedback $\gamma=R / M^{2}$, which gives only an indication of the actual feedback, is determined by the magnification $M$ and the reflectivity of the central area $R$ and is independent of the size of the reflecting area. $\theta_{d}$ is the full divergence angle based on the first minimum in the focus energy distribution, $E$ the output energy under matched discharge conditions, $\tau$ the pulse duration and $k$ the percentage of energy within the central peak.

Figure 6 shows the focus energy distributions for the three resonators with the same magnification $M=2.4$, mentioned in table 1 . These resonators are identical except for the reflectivity of the outcoupler. It is seen that the $72 \%$ spot reflectivity leads to an improved focus profile. Less energy (about $30 \%$ ) is lost in the side lobe. Further reduction to a spot reflectivity of $45 \%$ results in a focus that has a huge side lobe. $50 \%$ of the energy is lost in this side lobe. This is probably caused by the outcoupler: due to the coating used to obtain the reflectivity profile a phase difference between the central part of the beam and the beam edge will exist. This phase difference results in more side lobe energy. Interferometric measurements show that the $70 \%$ mirror has a phase difference of only $0.3 \pi$ and the $45 \%$ mirror a phase difference of $\pi$.

Reduction of the reflectivity of the outcoupler results in a lower output energy as can be found in table 1 . This is due to the lower feedback of the resonator, so that the output beam needs a longer build-up time, resulting 


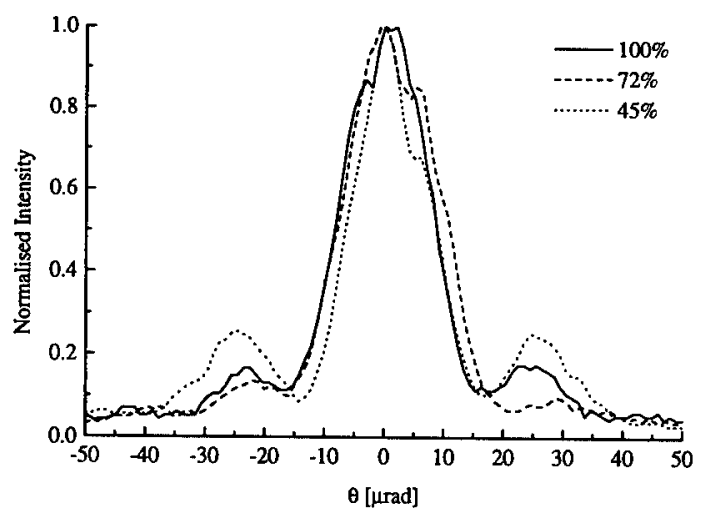

Figure 6. Focus energy distributions from resonators with different central reflectivities (100, 72 and $45 \%$ ). The magnification $M=2.4$ for all resonators

in a shorter pulse. To keep the geometrical feedback similar for different central reflectivity values the resonator magnification has to be decreased for decreasing mirror reflectance. Experiments have been performed using resonators having equal feedback (see table 1). To ensure a proper filling of the discharge volume the size of the reflecting spot on the outcoupling mirror was also increased. This however has a negative effect on the divergence as the focus from a resonator with a larger mirror size is also somewhat larger. The obtained results were similar to those obtained with the resonators having equal magnification mentioned above. The $72 \%$ reflectivity mirror shows an improvement and the $45 \%$ mirror a decrease of beam quality. The output energy is even somewhat higher for these two resonators.

\subsection{PHASE UNIFYING OUTCOUPLING MIRROR}

The transmission phase difference of the $45 \%$ mirror in the hard edge resonator experiments was nearly $\pi$. This phase difference can be decreased by using a special coating design: the phase unifying mirror [10, 11]. Experiments with such a mirror were performed with a resonator with magnification 1.6. Based on the coating a phase difference of only $0.16 \pi$ is expected. Interferometric measurements on this phase unifying mirror show only a very small phase shift which was hardly measurable. The results obtained with this mirror were much better than those obtained with the normal $45 \%$ mirror. Figure 7 shows the focus spots of the standard mirror res- 

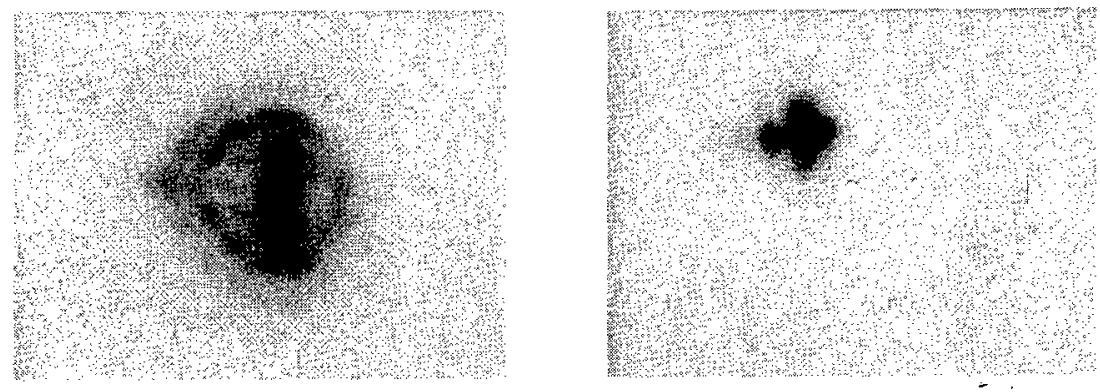

Figure 7. Focus spots from a hard edge resonator with a magnification of 1.6 and a central reflectance of $45 \%$ fitted with a standard mirror (left) and fitted with a phase unifying mirror (right)

TABLE 2. Characteristics of and experimental results obtained with the laser equipped with different gaussian unstable resonator configurations and operated under matched discharge conditions

\begin{tabular}{cccccccc}
$M$ & $R_{c}[\%]$ & $D_{r}[\mathrm{~mm}]$ & $\gamma[\%]$ & $\theta_{d}[\mu \mathrm{rad}]$ & $E[\mathrm{~mJ}]$ & $\tau[n s]$ & $k[\%]$ \\
\hline 2.4 & 65 & 8 & 11.3 & 25 & 412 & 144 & 43 \\
2.4 & 45 & 8 & 7.8 & 28 & 252 & 120 & 53 \\
2.4 & 17 & 8 & 3.0 & 27 & 212 & 115 & 55 \\
2.0 & 65 & 10 & 16.3 & 27 & 440 & 137 & 51 \\
2.0 & 45 & 10 & 11.3 & 37 & 342 & 129 & 64 \\
2.0 & 25 & 10 & 6.3 & 35 & 167 & 100 & 64 \\
1.6 & 45 & 12 & 17.6 & 50 & $\sim 200$ & $\sim 130$ & -
\end{tabular}

onator and the phase unifying resonator. The improvement is clear. The diffraction angle is comparable to the diffraction angle mentioned in table 1 for the $M=1.6$ resonator with $45 \%$ central reflectivity: $34 \mu \mathrm{rad}$, however approximately $80 \%$ of the $399 \mathrm{~mJ}$ output energy is found in the central peak, instead of less than $30 \%$ for the standard mirror.

\section{Gaussian unstable resonators}

A hard edge outcoupler will always result in a side lobe because of the diffraction at the hard edge. A focus spot without side lobes requires a soft edge outcoupling mirror. We used nearly gaussian mirrors because they can be analysed mathematically too. In table 2 the characteristics of the used gaussian resonators are given. $M$ is the resonator magnification, $R_{c}$ the central reflectivity of the gaussian reflection profile, $D_{r}$ is the $1 / \mathrm{e}^{2}$ diameter of this profile and $\gamma$ the feedback, which is given by $R_{c} / M^{2} . \theta_{d}$ is the full divergence angle based on the $1 / \mathrm{e}^{2}$ width of the focus profile. $E, \tau$ and 


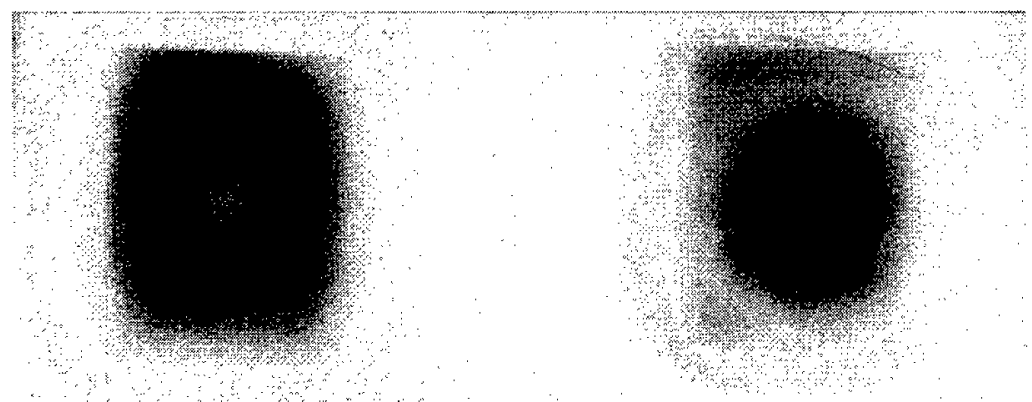

Figure 8 . The near field spots of two gaussian resonators. Left: $M=2.4$ and $R_{c}=65 \%$. Right: $M=2.0$ and $R_{c}=25 \%$

$k$ are the pulse energy, pulse duration and energy content within the full divergence angle respectively.

We used the same magnifications as we used for the hard edge unstable resonator. This choice was based on the good results we obtained with those resonators. We used different reflectivities to be able to see the influence on the beam. The high central reflectivity results in a beam with a smooth edged central hole in the near field as can be seen in figure 8 . The energy from this resonator is quite high (412 $\mathrm{mJ}$ under almost matched discharge conditions) due to good filling of the discharge volume. Figure 8 also shows the near field of a low central reflectivity resonator. It was designed to have a maximally flat near field. The condition for this is [7]

$$
R_{c} M^{2}=1
$$

The energy from this resonator is rather low $(167 \mathrm{~mJ})$ because the discharge volume is not properly filled and the optical pulse is shorter (100 ns) due to the low feedback. The beam is flat topped as expected.

It can be seen from figure 9 that the central hole in the near field of the $M=2.4$ and $R_{c}=65 \%$ still leads to a small side lobe. Calculations show that this is in accordance with theory. Even from the maximally flat resonator $\left(M=2.0\right.$ and $\left.R_{c}=25 \%\right)$ still a very small side lobe is expected from theory. The experiments show a slightly wider focus with a low but wide shoulder as shown in figure 9 . This shoulder results in a lower energy content within the $1 / e^{2}$ diameter $\left(\theta_{d}\right)$. In table 2 the energy content within the divergence angle is given for the gaussian resonators. For the $M=2.0$ and $R_{c}=25 \%$ resonator an energy content of $84 \%$ is expected from theory and the experiments resulted in $64 \%$. The calculated divergence angle is $21 \mu \mathrm{rad}$ and the experimental value is $35 \mu \mathrm{rad}$, thus resulting in a value for the TDL parameter of 1.8 . 

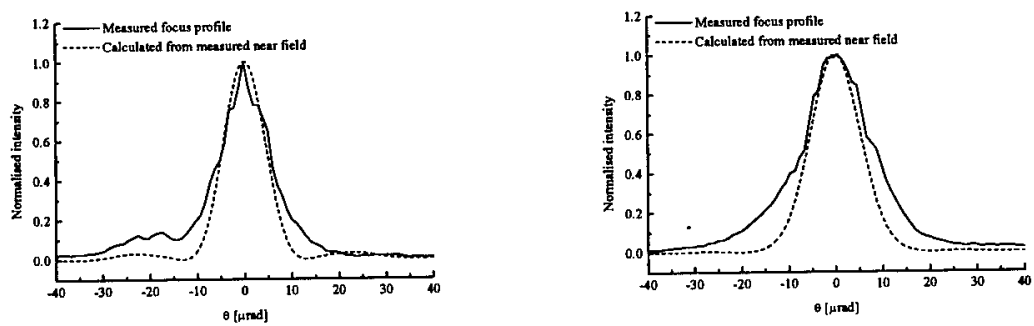

Figure 9. Measured and calculated focus energy distributions for two gaussian resonators. Left: $M=2.4$ and $R_{c}=65 \%$. Right: $M=2.0$ and $R_{c}=25 \%$

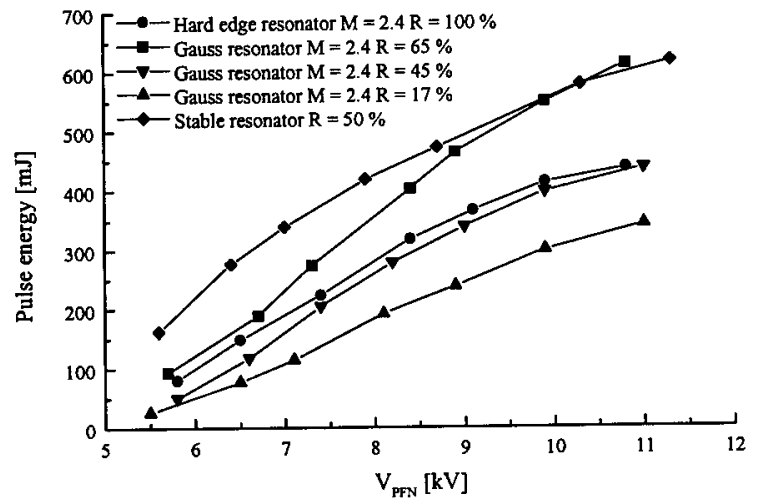

Figure 10. Output energy as a function of the sustainer voltage for different resonators

\section{Performance comparison}

In the previous sections we only looked at the divergence and the beam profiles. In this section a comparison will be made between the resonators with respect to output energy, pulse duration, average power and brightness.

Figure 10 shows the output energy of different resonators as a function of the sustainer voltage. It is seen that except for the highest sustainer voltage the highest output can be obtained with a stable resonator. The hard edge resonator shows an output energy reduction of about $20 \%$ compared to the stable resonator. With the high central reflectivity gauss resonator it is possible to extract a similar amount of energy from the gain medium as with the stable resonator, especially for higher sustainer voltages. However, the 


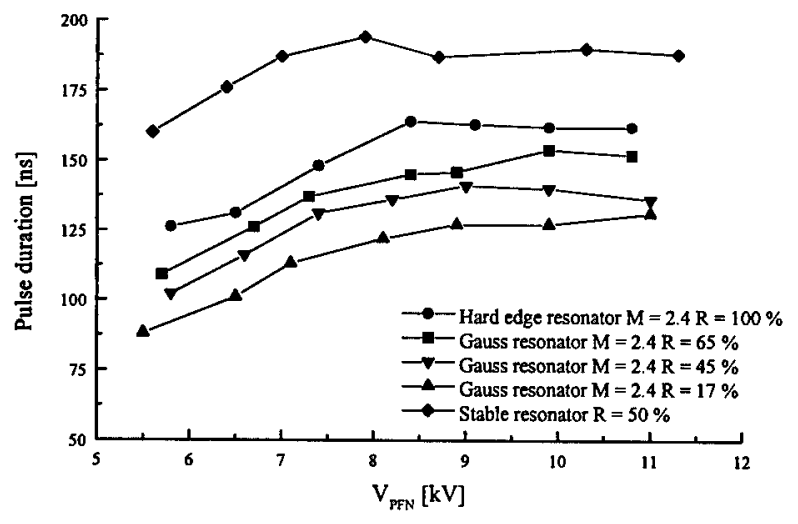

Figure 11. Pulse duration (FWHM) as a function of the sustainer voltage for different resonators

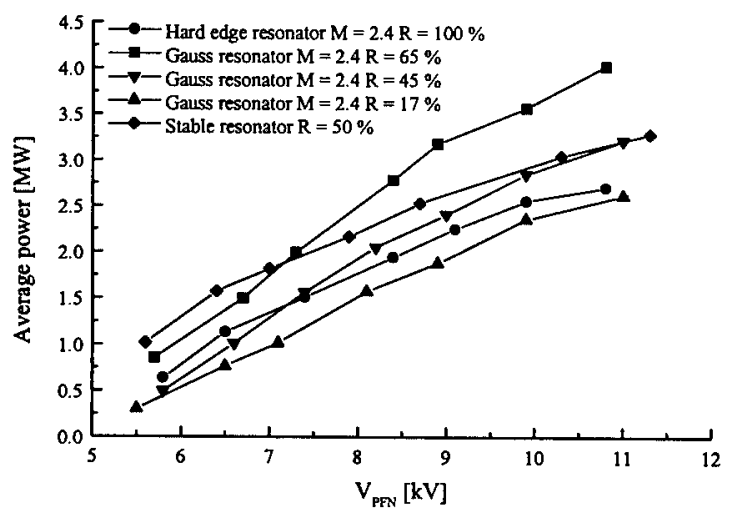

Figure 12. Average pulse power as a function of the sustainer voltage for different resonators

pulse duration is much shorter for the gaussian resonators as can be seen from figure 11. This pulse duration is even shorter than the pulse duration from the hard edge unstable resonator. This results in the highest average pulse powers, which is defined by the energy divided by the pulse duration $(=E / \tau)$, for the high central reflectivity gaussian resonator as seen in figure 12.

The brightness of the beam is a convenient parameter to compare the 
output from the different resonators. The brightness can be defined as

$$
B=k \frac{E}{\tau}\left(\frac{1}{\pi \frac{\theta_{d}}{2} \frac{M D_{x}}{2}}\right)^{2}
$$

Under matched discharge conditions, $V_{\mathrm{PFN}}=8 \mathrm{kV}$, we obtained from the HR hard edge resonator a brightness of $5.5^{*} 10^{14} \mathrm{~W} /\left(\mathrm{cm}^{2} \cdot \mathrm{sr}\right)$. This is an enourmous increase compared to the brightness from the stable planoconcave resonator: $\mathrm{B}=1.5^{*} 10^{10} \mathrm{~W} /\left(\mathrm{cm}^{2} \cdot \mathrm{sr}\right)$. This could be futher increased to $1.2^{*} 10^{15} \mathrm{~W} /\left(\mathrm{cm}^{2} \cdot \mathrm{sr}\right)$ if we pushed the discharge to its limits. With the phase unifying resonator a slightly larger brightness could be obtained: $7.0^{*} 10^{14} \mathrm{~W} /\left(\mathrm{cm}^{2} \cdot \mathrm{sr}\right)$ under matched discharge conditions and $1.4^{*} 10^{15} \mathrm{~W} /\left(\mathrm{cm}^{2} \cdot \mathrm{sr}\right)$ maximally. The gaussian resonator with $M=2.0$ and $R_{c}=65 \%$ resulted in a brightness of $9.1^{*} 10^{14} \mathrm{~W} /\left(\mathrm{cm}^{2} \cdot \mathrm{sr}\right)$ under matched discharge conditions. Hence under matched discharge conditions the best results are obtained with the gaussian resonator.

\section{Conclusions}

In this paper we presented results from measurements on different resonator designs for a long pulse, low gain $\mathrm{XeCl}$ excimer laser. A stable plano concave resonator with $50 \%$ feedback results in a homogeneous nearly square beam with $500 \mathrm{~mJ}$ output energy and $7 \mathrm{mrad}$ divergence under matched discharge conditions. With a high reflectivity hard edge resonator having a magnification of 2.4 a 1.3 times diffraction limited beam can be obtained. This beam has under matched discharge conditions an output energy of about $340 \mathrm{~mJ}$, a divergence of $32 \mu \mathrm{rad}$ and a brightness of $5.5^{*} 10^{14} \mathrm{~W} /\left(\mathrm{cm}^{2} \cdot \mathrm{sr}\right)$. The energy content of this beam within the divergence angle is about $60 \%$. We have shown that this can be improved by using partial reflecting hard edge outcouplers. Attention has to be paid to the phase distortion by the outcoupler. This can be diminished by using a phase unifying outcoupler. This resonator resulted in a beam with about $400 \mathrm{~mJ}$ output energy, $34 \mu \mathrm{rad}$ divergence, an energy content of about $80 \%$ and a brightness of $7.0^{*} 10^{14} \mathrm{~W} /\left(\mathrm{cm}^{2} \cdot \mathrm{sr}\right)$.

To reduce the side lobe energy further soft edge mirrors have to be used. We have shown that with a resonator having a magnification of 2.0 and a central reflectivity of $65 \%$ a beam with $440 \mathrm{~mJ}$ energy and $27 \mu \mathrm{rad}$ divergence can be obtained under matched discharge conditions. The brightness of this beam is $9.1^{*} 10^{14} \mathrm{~W} /\left(\mathrm{cm}^{2} \cdot \mathrm{sr}\right)$. Thus the best results were obtained using gaussian resonators. 


\section{Acknowledgements}

The present work has been supported by the Netherlands Technology Foundation (STW). The authors would like to thank M.J. Zwegers from the Nederlands Centrum voor Laser Research (NCLR) for performing part of the measurements and J. van Hespen en T. van Drunick from the optics workshop at the Applied Physics department of Twente University for supplying us with the special mirrors used for the experiments presented in this paper.

\section{References}

1. F.A. van Goor, W.J. Witteman, J.C.M. Timmermans, J. van Spijker, and J. Couperus. High-average power xecl laser with x-ray pre-ionization and spiker-sustainer excitation. In M. Bohrer, T. Letardi, D. Schuöcker, and H. Weber, editors, HighPower Gas and Solid State Lasers, volume 2206 of Proceedings Europto Series, pages 30-40. SPIE, 1994.

2. T.J. McKee. Optical cavity design for long pulse excimer lasers. Applied Optics, 30(6):635-644, 1991.

3. T.J. McKee and S. Fendrykowski. Long-pulse excimer laser with a variable reflectivity mirror resonator. Applied Optics, 32(3):275-277, 1993.

4. S.E. Kovalenko, V. Losev, and M.R. Perrone. Super-gaussian resonators for long pulse xecl lasers. Applied Optics, 33(18):4082-4086, 1994.

5. J.C.M. Timmermans. Double discharge XeCl-laser. $\mathrm{PhD}$ thesis, University of Twente, Enschede, the Netherlands, 1995.

6. J.W. Gerritsen, A.L. Keet, G.J. Ernst, and W.J. Witteman. High-efficiency operation of a gas discharge xecl laser using a magnetically induced resonant voltage overshoot circuit. Journal of Applied Physics, 67(7):3517-3519, 1990.

7. A.E. Siegman. Lasers. University Science Books, 20 Edgehill Road, Mill Valley, CA 94941, USA, 1986.

8. P.E. Dyer. Unstable resonators. In D.R. Hall and P.E. Jackson, editors, The physics and technology of laser resonators, chapter 2, pages 21-39. Institute of Physics Publishing, Techno House, Redcliffe Way, Bristol BS1 6NX, England, 1992.

9. S. Bollanti, P. di Lazarro, and D. Murra. How many times is a beam diffraction limited? Optics Communications, 134(1-6):503-513, 1997.

10. V.B. Kaul', S.V. Mel'chenko, M.R. Perrone, A. Piegari, and V.F. Tarasenko. Near diffraction limited output from a $100 \mathrm{~ns}$ xecl laser fitted with a phase unifying cavity. Journal of Modern Optics, 42(11):2229-2238, 1995.

11. M.R. Perrone. On the performance of a phase-unifying unstable resonator for excimer lasers. Optics Communications, 116(1-3):101-108, 1995. 